\title{
Asleep or awake motor mapping for resection of perirolandic glioma in the nondominant hemisphere? Development and validation of a multimodal score to tailor the surgical strategy
}

\author{
Marco Rossi, MD, 1,2 Guglielmo Puglisi, PhD, 2,3 Marco Conti Nibali, MD,1,2 Luca Viganò, PhD,,2 \\ Tommaso Sciortino, MD, ${ }^{1,2}$ Lorenzo Gay, MD, ${ }^{1,2}$ Antonella Leonetti, PhD, ${ }^{2,3}$ Paola Zito, MD, ${ }^{4}$ \\ Marco Riva, MD, ${ }^{2,3}$ and Lorenzo Bello, MD ${ }^{1,2}$
}

\begin{abstract}
${ }^{1}$ Neurosurgical Oncology Unit, Department of Oncology and Hemato-Oncology, and ${ }^{3}$ Neurosurgical Oncology Unit, Department of Medical Biotechnologies and Translational Medicine, Università degli Studi di Milano; ${ }^{2}$ Neurosurgical Oncology Unit, IRCCS Istituto Ortopedico Galeazzi, Milano; and ${ }^{4}$ Department of Anesthesia and Intensive Care, Humanitas Research Hospital, IRCCS, Rozzano, Italy
\end{abstract}

OBJECTIVE Resection of glioma in the nondominant hemisphere involving the motor areas and pathways requires the use of brain-mapping techniques to spare essential sites subserving motor control. No clear indications are available for performing motor mapping under either awake or asleep conditions or for the best mapping paradigm (e.g., resting or active, high-frequency [HF] or low-frequency [LF] stimulation) that provides the best oncological and functional outcomes when tailored to the clinical context. This work aimed to identify clinical and imaging factors that influence surgical strategy (asleep motor mapping vs awake motor mapping) and that are associated with the best functional and oncological outcomes and to design a "motor mapping score" for guiding tumor resection in this area.

METHODS The authors evaluated a retrospective series of patients with nondominant-hemisphere glioma-located or infiltrating within $2 \mathrm{~cm}$ anteriorly or posteriorly to the central sulcus and affecting the primary motor cortex, its fibers, and/ or the praxis network-who underwent operations with asleep (HF monopolar probe) or awake (LF and HF probes) motor mapping. Clinical and imaging variables were used to design a motor mapping score. A prospective series of patients was used to validate this motor mapping score.

RESULTS One hundred thirty-five patients were retrospectively analyzed: 69 underwent operations with asleep (HF stimulation) motor mapping, and 66 underwent awake (LF and HF stimulation and praxis task evaluation) motor mapping. Previous motor (strength) deficit, previous treatment (surgery/radiotherapy), tumor volume $>30 \mathrm{~cm}^{3}$, and tumor involvement of the praxis network (on MRI) were identified and used to design the mapping score. Motor deficit, previous treatment, and location within or close to the central sulcus favor use of asleep motor mapping; large tumor volume and involvement of the praxis network favor use of awake motor mapping. The motor mapping score was validated in a prospective series of 52 patients-35 underwent operations with awake motor mapping and 17 with asleep motor mapping on the basis of the score indications - who had a low rate of postoperative motor-praxis deficit (3\%) and a high extent of resection (median $97 \%$; complete resection in $>70 \%$ of patients).

CONCLUSIONS Extensive resection of tumor involving the eloquent areas for motor control is feasible, and when an appropriate mapping strategy is applied, the incidence of postoperative motor-praxis deficit is low. Asleep (HF stimulation) motor mapping is preferable for lesions close to or involving the central sulcus and/or in patients with preoperative strength deficit and/or history of previous treatment. When a patient has no motor deficit or previous treatment and has a lesion (> $30 \mathrm{~cm}^{3}$ ) involving the praxis network, awake mapping is preferable.

https://thejns.org/doi/abs/10.3171/2020.11.JNS202715

KEYWORDS functional balance; motor mapping; apraxia; glioma; awake craniotomy; intraoperative neurophysiology; oncology

ABBREVIATIONS DWI = diffusion-weighted imaging; EMG = electromyography; $E O R=$ extent of resection; $H F=$ high-frequency; hMT = hand-manipulation task; LF = lowfrequency; $\mathrm{MEP}=$ motor evoked potential; $\mathrm{MNI}=$ Montreal Neurological Institute; $\mathrm{MRC}=$ Medical Research Council; RTV = residual tumor volume; SMA = supplementary motor area.

SUBMITTED July 31, 2020. ACCEPTED November 2, 2020.

INCLUDE WHEN CITING Published online June 18, 2021; DOI: 10.3171/2020.11.JNS202715. 
I $\mathrm{N}$ brain tumor surgery, preservation of motor function is critical because of the limited chance of improvement when severe postoperative motor deficits occur. ${ }^{1-4}$ Motor function varies in complexity, ranging from simple or multiple muscle contraction to the ability to learn a complex motor skill. Physiologically, a motor action requires the interaction of the primary motor area with the surrounding networks. ${ }^{5-8}$ Brain-mapping techniques allow identification and preservation of essential cortical and subcortical sites during surgery. The surgeon adapts the mapping strategy to the circuit(s) and motor functions that need to be explored. ${ }^{9}$ Traditionally, two different motor mapping strategies are applied: a resting approach (mostly performed on asleep patients), in which, without any patient collaboration, direct electrical stimulation is applied over the investigated site and the obtained motor response is evaluated either on the basis of overt movement or with electromyography (EMG); or a collaborative approach, in which the patient is awake and asked to perform a motor task while the observed interference during stimulation is evaluated.,10,11 These two approaches allow the surgeon to map different components of the complex network involved in motor control. The resting-asleep setting identifies the primary motor cortex and its descending fibers with high specificity and sensitivity; ${ }^{12}$ the collaborativeawake setting mainly investigates the nonprimary motor areas and related circuitry. ${ }^{13-16}$

It is common knowledge that an awake phase is recommended for dominant-hemisphere tumor resection in order to ensure safe resection and preservation of language and cognitive functions; in this context, it is possible to extend mapping to higher motor cognitive functions. ${ }^{17} \mathrm{On}$ the contrary, when a tumor develops in the nondominant hemisphere, the decision to perform motor mapping under awake or asleep anesthesia is, at the moment, based on surgeon preference and/or experience. In addition, most available studies have evaluated motor outcome after tumor removal and report just the effect on motor strength, which is only a small portion of global motor capacity. ${ }^{1,18-20}$ For instance, apraxia could occur, in the absence of motor strength deficit, when a tumor located within $2 \mathrm{~cm}$ of the primary motor area is removed and the praxis network is not carefully tested intraoperatively with dedicated tasks. ${ }^{14,21}$ Furthermore, it is still unknown which clinical or tumor imaging factors favor asleep versus awake motor mapping. In fact, no clear indication is available for patients with glioma in the nondominant hemisphere, and there is also a lack of general consensus on the intraoperative anesthesia mapping paradigm that provides the best oncological and functional outcomes when tailored to the clinical context. ${ }^{22}$

In recent years, we have operated on patients with tumor in the nondominant hemisphere under either asleep or awake conditions, with those in the latter group performing a hand-manipulation task (hMT) to evaluate the praxis network. ${ }^{14}$ In this work, first we report the functional and oncological data of a retrospective series of patients treated for tumor located within $2 \mathrm{~cm}$ anteriorly or posteriorly to the central sulcus in the nondominant hemisphere and affecting the primary motor cortex, its fibers, and/or praxis network. Patients were classified as "asleep" or "awake" according to the adopted motor mapping strategy. With this analysis, we aimed to identify which clinical or imaging features influence surgical strategy and functional and oncological outcome (in terms of extent of resection [EOR]). Based on these findings, a "motor mapping score" was generated to serve as a guide for choosing the most appropriate motor mapping strategy. The score was then applied to a prospective consecutive series of patients with comparable clinical features; again, great attention was paid to functional and oncological outcomes. The goal of this study was to provide suggestions to help surgeons decide to use asleep or awake motor mapping when approaching a tumor located within $2 \mathrm{~cm}$ of the primary motor cortex.

\section{Methods}

\section{Patients and Study Design}

Patients who fulfilled the following inclusion criteria were included: a) tumor in the right nondominant hemisphere; b) tumor location within $2 \mathrm{~cm}$ of the central sulcus; c) surgery performed with either intraoperative asleep or awake anesthesia; d) availability of full clinical and radiological data; and e) availability of neuropsychological evaluation data, inclusive of praxis function. $\mathrm{Pa}-$ tients were categorized into two groups (Fig. 1 and Table 1). 1) The retrospective series included all patients consecutively treated at our ward between January 2014 and July 2018 (Humanitas Research Hospital, Rozzano, Italy). This series was used to identify the clinical and imaging variables needed to develop the "motor mapping score." 2) The prospective series enrolled all patients with tumors in similar locations who were prospectively admitted to our ward from August 2018 to December 2019 (Humanitas Research Hospital). For these patients, the motor mapping score determined the motor mapping strategy that was applied. All patients gave informed consent to the procedure.

\section{Neurological and Neuropsychological Evaluations}

Patients were evaluated before surgery and 5 days and 1-3 months after surgery. Neurological evaluation of motor (strength) function used the Medical Research Council (MRC) grading system. Seizure history and preoperative and postoperative seizure control were also recorded.

At the same time points, neuropsychological examination was performed, inclusive of the ideomotor-apraxia task, to detect subtle praxis deficits. The test, based on imitations of hand gestures, is considered the gold standard for detecting apraxia. A neuropsychologist asked the patient to imitate 10 intransitive gestures (use of objects was not required), and the patient was instructed to use both the ipsilateral and contralateral arms of the affected hemisphere. ${ }^{14,17}$ No verbal description of the movements to be imitated was provided. When a gesture was not reproduced correctly on the first demonstration, a second one was allowed. Hand apraxia was determined to be present when the patient obtained a pathological score $(<2)$.

\section{Imaging Analysis}

The preoperative MRI protocol, acquired on a 3.0-T 


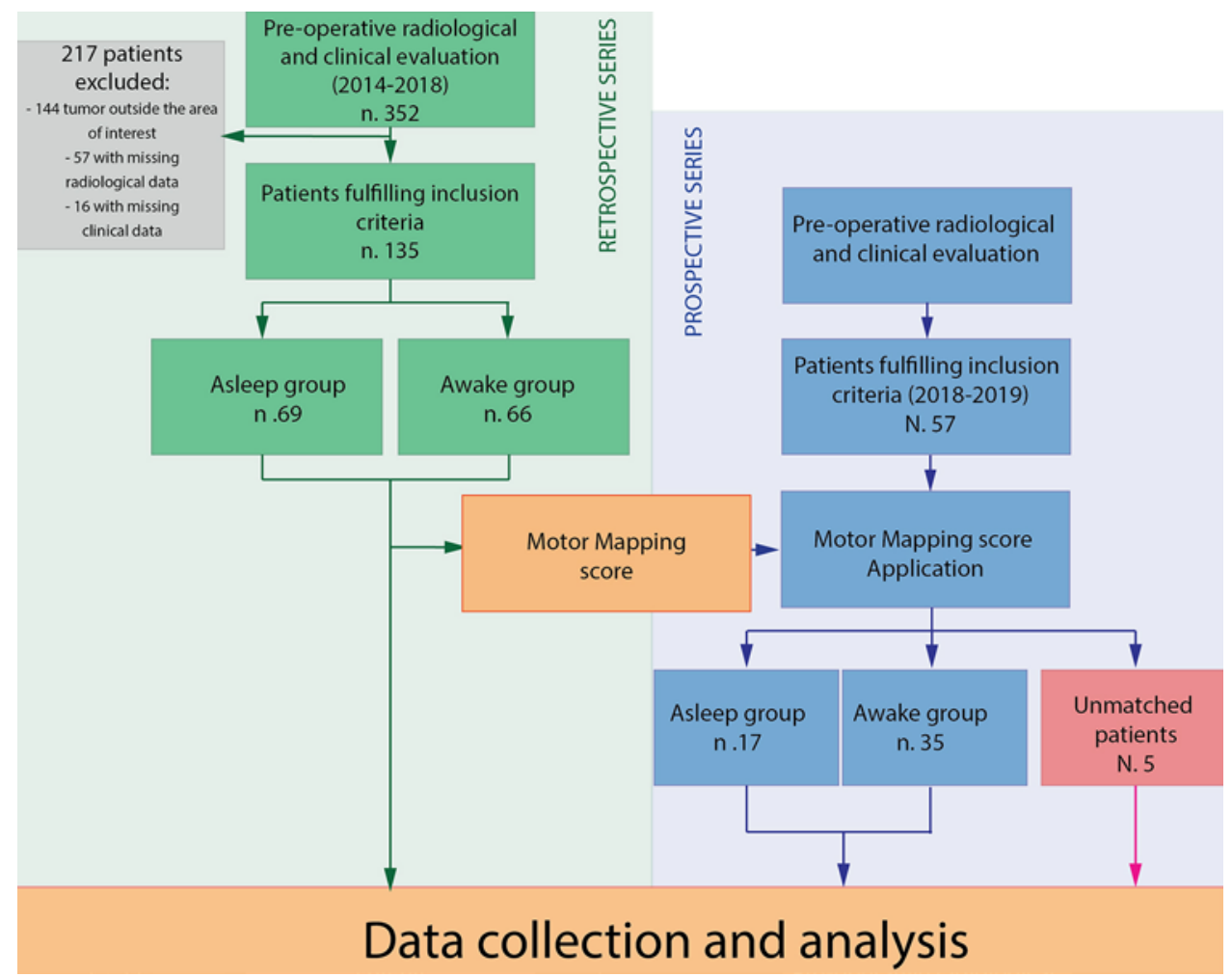

FIG. 1. Diagram showing the study design. In both the retrospective and prospective series, patients who fulfilled the inclusion criteria were categorized according to the adopted anesthesia mapping protocol as asleep or awake. The unmatched patient group in the prospective series included those patients for whom the adopted anesthesia mapping protocol differed from that indicated by the mapping score. Figure is available in color online only.

Magnetom Verio system (Siemens), included a) axial 3DFLAIR imaging; b) post-Gd 3D T1-weighted imaging; and c) diffusion-weighted imaging (DWI) and apparent diffusion coefficient imaging. Patients underwent both immediate (within 48 hours) and 2-month postoperative MRI (volumetric FLAIR and post-Gd T1-weighted imaging) to estimate EOR. ${ }^{23}$ Immediate postoperative diffusion-weighted MRI was performed to evaluate ischemia. Preoperative FLAIR and post-Gd T1-weighted imaging were used for spatial analysis of tumor location.

\section{Surgical Procedure}

Resection was always performed according to functional boundaries. Brain-mapping and brain-monitoring techniques were applied. In all cases, neuronavigation was available for surgical planning and intraoperative use to locate the tumor site and boundaries. However, resection was guided with intraoperative motor mapping. For patients in the retrospective series, the motor mapping strategy and related anesthesia setting (asleep vs asleepawake-asleep anesthesia) were decided by the surgeon (L.B. and M. Rossi) on the basis of the findings of previous work..$^{1,12,14}$ All procedures (asleep or awake) were performed by the same surgical team, which included the first (M. Rossi) and senior (L.B.) authors. In the prospective cohort, the type of mapping was determined with the motor mapping score.
Type and percentage of postoperative complications and perioperative deaths were recorded as well.

\section{Brain Monitoring}

EEG and ECoG were continuously used to monitor the depth of anesthesia and occurrence of seizures/afterdischarges. Free-running EMG activity was monitored from the beginning of surgery with a multichannel recording setup, and as many as 24 electromyograms were recorded from different contralateral and ipsilateral muscles. ${ }^{1}$ To monitor the integrity of the descending motor pathways throughout the procedure, the recording system was used to monitor 1) free-running background EMG activity, 2) motor responses to brain-mapping stimulation, and 3) motor evoked potentials (MEPs) due to stimulation of M1 with the train-of-five technique. ${ }^{12}$ MEPs were recorded with transcranial electrodes from incision to closure, and with strip electrodes during resection; small 4-contact strips were placed very close to the surgical field.

\section{Brain Mapping and Resection \\ Asleep Setting}

For patients undergoing surgery in the asleep setting, motor mapping was performed with the high-frequency (HF) technique (500-msec monophasic stimulus with 3-msec interstimulus interval, delivered with a monopo- 
TABLE 1. Clinical and imaging variables of the patients included in the retrospective and prospective series

\begin{tabular}{|c|c|c|c|}
\hline Variable & $\begin{array}{l}\text { Retrospective } \\
\quad(n=135)\end{array}$ & $\begin{array}{l}\text { Prospective } \\
\quad(n=52)\end{array}$ & $\mathrm{p}$ Value \\
\hline \multicolumn{4}{|l|}{ Preop } \\
\hline \multicolumn{4}{|l|}{$\begin{array}{l}\text { Anesthesia mapping } \\
\text { protocol }\end{array}$} \\
\hline Awake & $66(49)$ & $35(67)$ & \multirow{2}{*}{0.241} \\
\hline Asleep & $69(51)$ & $17(33)$ & \\
\hline \multicolumn{4}{|l|}{ Sex } \\
\hline Male & $73(54)$ & $34(65)$ & \multirow{2}{*}{0.161} \\
\hline Female & $62(46)$ & $18(35)$ & \\
\hline Age, mean (range), yrs & $44.2(18-75)$ & $44.0(20-74)$ & 0.937 \\
\hline \multicolumn{4}{|l|}{ 1st surgery } \\
\hline Yes & $84(62)$ & $31(60)$ & \multirow{2}{*}{0.743} \\
\hline No & $51(38)$ & $21(40)$ & \\
\hline RT & $14(10)$ & $9(17)$ & 0.196 \\
\hline \multicolumn{4}{|l|}{ Seizure } \\
\hline Yes & $107(79)$ & $41(79)$ & \multirow{2}{*}{0.784} \\
\hline No & $28(21)$ & $11(21)$ & \\
\hline \multicolumn{4}{|l|}{ Seizure control } \\
\hline Yes & $83(78)$ & $35(85)$ & \multirow{2}{*}{0.277} \\
\hline No & $24(22)$ & $6(15)$ & \\
\hline \multicolumn{4}{|l|}{ MRC grade } \\
\hline $5 / 5$ & $112(83)$ & $49(94)$ & \multirow{3}{*}{0.244} \\
\hline $4 / 5$ & $18(13)$ & $3(6)$ & \\
\hline$\leq 3 / 5$ & $5(4)$ & $0(0)$ & \\
\hline Hand apraxia & $3(2)$ & $1(2)$ & 0.317 \\
\hline \multicolumn{4}{|l|}{ Lobe } \\
\hline Frontal & $106(78)$ & $34(65)$ & \multirow{4}{*}{0.099} \\
\hline Parietal & $18(13)$ & $15(29)$ & \\
\hline Frontoparietal & $6(5)$ & $1(2)$ & \\
\hline Other & $5(4)$ & $2(4)$ & \\
\hline \multicolumn{4}{|l|}{ Tumor vol, $\mathrm{cm}^{3}$} \\
\hline Mean (range) & $39.7(1.4-159)$ & $49.4(1-229)$ & \multirow{2}{*}{0.003} \\
\hline Median & 25.2 & 26.2 & \\
\hline \multicolumn{4}{|l|}{$\begin{array}{l}\text { Praxis network } \\
\text { involvement }\end{array}$} \\
\hline Yes & $67(50)$ & $30(58)$ & \multirow{2}{*}{0.130} \\
\hline No & $68(50)$ & $22(42)$ & \\
\hline \multicolumn{4}{|l|}{ SMA involvement } \\
\hline Yes & $27(20)$ & $2(4)$ & \multirow{2}{*}{0.006} \\
\hline No & $108(80)$ & $50(96)$ & \\
\hline Periop morbidity* & $2(1)$ & $1(3)$ & 0.323 \\
\hline \multicolumn{4}{|l|}{ Postop } \\
\hline \multicolumn{4}{|l|}{ MRC grade } \\
\hline \multicolumn{4}{|l|}{ At 5 days } \\
\hline $5 / 5$ & $54(40)$ & $32(61)$ & \multirow{3}{*}{0.013} \\
\hline $4 / 5$ & $53(39)$ & $12(23)$ & \\
\hline$\leq 3 / 5$ & $28(21)$ & $8(16)$ & \\
\hline
\end{tabular}

CONTINUED IN NEXT COLUMN »
》 CONTINUED FROM PREVIOUS COLUMN

TABLE 1. Clinical and imaging variables of the patients included in the retrospective and prospective series

\begin{tabular}{|c|c|c|c|}
\hline Variable & $\begin{array}{l}\text { Retrospective } \\
\quad(n=135)\end{array}$ & $\begin{array}{c}\text { Prospective } \\
(n=52)\end{array}$ & $p$ Value \\
\hline \multicolumn{4}{|c|}{ MRC grade (continued) } \\
\hline \multicolumn{4}{|l|}{ At $1-3$ mos } \\
\hline $5 / 5$ & $110(81)$ & $46(88)$ & \multirow{3}{*}{0.494} \\
\hline $4 / 5$ & $22(16)$ & $5(10)$ & \\
\hline$\leq 3 / 5$ & $2(3)$ & $1(2)$ & \\
\hline \multicolumn{4}{|l|}{ Apraxia } \\
\hline \multicolumn{4}{|l|}{ At 5 days } \\
\hline Yes & $21(16)$ & $8(15)$ & \multirow{2}{*}{0.977} \\
\hline No & $114(84)$ & $44(85)$ & \\
\hline \multicolumn{4}{|l|}{ At $1-3$ mos } \\
\hline Yes & $12(10)$ & $2(4)$ & \multirow{2}{*}{0.231} \\
\hline No & $121(90)$ & $50(96)$ & \\
\hline \multicolumn{4}{|l|}{ SMA syndrome } \\
\hline Yes & $4(15)$ & $0(0)$ & \multirow{2}{*}{0.963} \\
\hline No & $23(85)$ & $2(100)$ & \\
\hline \multicolumn{4}{|l|}{ Engel class } \\
\hline I & $94(70)$ & $48(92)$ & \multirow{4}{*}{0.015} \\
\hline II & $31(23)$ & $4(8)$ & \\
\hline III & $3(2)$ & $0(0)$ & \\
\hline IV & $2(2)$ & $0(0)$ & \\
\hline \multicolumn{4}{|c|}{ Residual tumor vol, $\mathrm{cm}^{3}$} \\
\hline Mean (range) & $2.3(0-53)$ & $1.7(0-19)$ & \multirow{2}{*}{0.643} \\
\hline Median & 0 & 0 & \\
\hline \multicolumn{4}{|l|}{ EOR } \\
\hline Mean, $\%$ & 95 & 97 & 0.337 \\
\hline $100 \%$ & $99(73)$ & $37(71)$ & \\
\hline $90-99 \%$ & $16(12)$ & $13(25)$ & \\
\hline$<90 \%$ & $20(15)$ & $2(4)$ & 0.264 \\
\hline \multicolumn{4}{|l|}{ Alteration on DWI } \\
\hline Eloquent site & $20(15)$ & $3(6)$ & \multirow{3}{*}{0.435} \\
\hline Not eloquent site & $23(17)$ & $13(23)$ & \\
\hline No & $92(68)$ & $36(71)$ & \\
\hline \multicolumn{4}{|l|}{ Histologic diagnosis } \\
\hline LGG & $48(54)$ & $34(65)$ & \multirow{3}{*}{0.007} \\
\hline HGG & $73(35)$ & $16(31)$ & \\
\hline Other† & $14(10)$ & $2(4)$ & \\
\hline \multicolumn{4}{|l|}{ RT } \\
\hline Yes & $62(46)$ & $17(33)$ & \multirow{2}{*}{0.163} \\
\hline No & $73(54)$ & $35(67)$ & \\
\hline
\end{tabular}

HGG = high-grade glioma; $L G G$ = low-grade glioma; $R T$ = radiotherapy. Values are shown as number (percent) unless indicated otherwise. Boldface type indicates statistical significance $(p<0.05)$.

* In the retrospective series, 1 patient died of pulmonary embolism, and 1 patient completely recovered from a postoperative hematoma that was immediately evacuated. In the prospective series, 1 patient died of pulmonary embolism.

† Includes 6 patients with metastasis, 4 with cortical dysplasia, 4 with cavernous malformation, and 2 with gliosis. 
lar probe). After opening the dura mater, cortical mapping was used to identify the primary motor cortex, where a strip electrode was placed for continuous MEP monitoring at the cortical motor threshold. Hence, cortical motor mapping was used to identify the safe entry zone where corticectomy was performed. Next, HF stimulation was continuously applied at the subcortical level to identify the M1 originating fibers as the functional boundary of resection, until a subcortical motor threshold of $3 \mathrm{~mA}$ was reached. ${ }^{12}$ To ensure continuous subcortical mapping, the assistant surgeon placed and kept the monopolar probe in the surgical field very close to the site of resection, thus detecting any motor response. When a motor response was identified, a current-intensity curve was obtained to estimate the distance from the M1 originating fibers. Resection was continued until a subcortical motor threshold of $3 \mathrm{~mA}$ was reached.

\section{Awake Setting}

In this setting, along with identification and preservation of the M1 fibers with HF stimulation (as described above), patients were asked to perform hMT, with low-frequency (LF) stimulation (1-msec, 60-Hz biphasic stimulation, delivered with a bipolar probe) used to identify the praxis circuit. Patients were awakened for this purpose. The lowest current intensity that interfered with hMT execution was applied throughout the task. At the cortical level, sites identified with either hMT or HF stimulation (resting condition) were preserved. At the subcortical level, sites identified with both hMT (praxis circuit) and HF stimulation (resting condition) were considered the functional boundaries where resection should stop. ${ }^{14}$ Again, to ensure continuous subcortical mapping and detection of any motor interference or response, the bipolar (for LF stimulation) or monopolar (for HF stimulation) probes were placed and kept in the surgical field very close to the site of resection by the assistant surgeon. For LF stimulation, the same current intensity that had been established cortically was delivered at the subcortical level. For HF stimulation, the same protocol described for asleep motor mapping was used.

\section{Extent of Resection}

EOR was calculated on 48-hour (volumetric post-Gd T1-weighted images for enhancing lesions [target of resection]) or 2-month (volumetric FLAIR for nonenhancing lesions [target of resection]) postoperative MR images. FLAIR-hyperintense or T1-weighted Gd-enhanced signal abnormalities were included in the lesion load for noncontrast-enhancing lesion or high-grade glioma, respectively, and were reported in cubic centimeters. ${ }^{23}$ Volumes were calculated with Elements SmartBrush software (Brainlab) by four blinded investigators (M. Rossi, L.G., M.C.N., and T.S.). EOR was classified according to the percentage of resection and residual tumor volume (RTV). The first corresponds to percentage of volume resected with respect to preoperative volume ([preoperative volume - postoperative volume]/preoperative volume) and was classified as gross-total resection $(\mathrm{EOR}=100 \%)$, near-total resection (EOR > 90\%-99\%), or subtotal resection (EOR < 90\%). The volume of any residual abnormality was measured and reported in cubic centimeters.
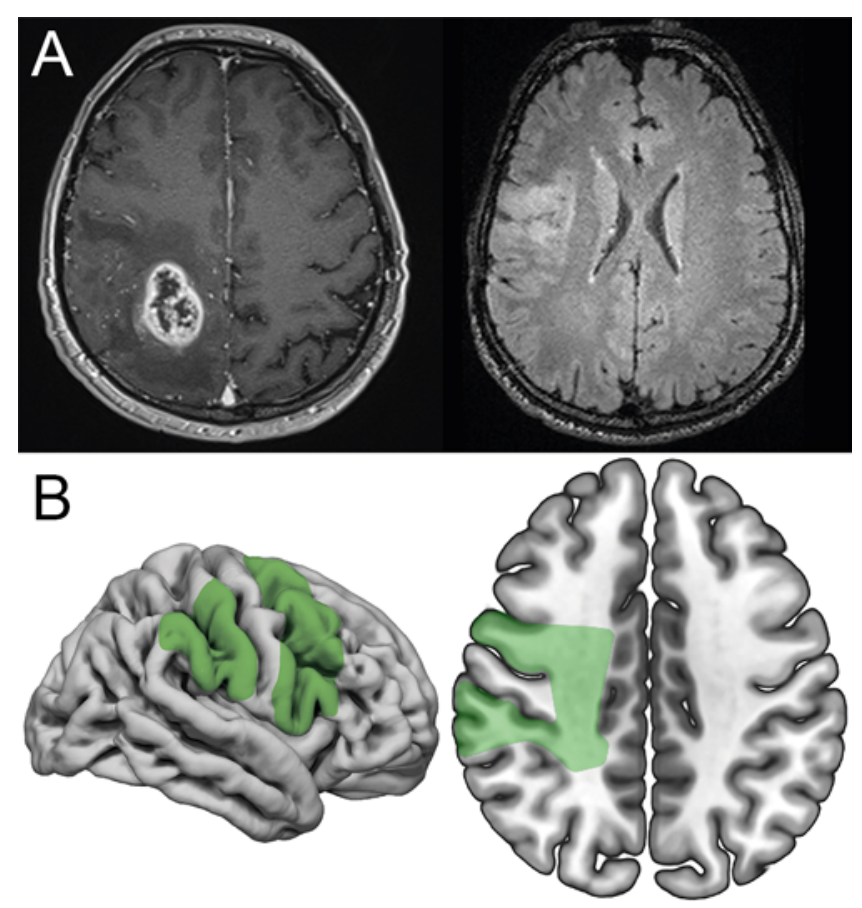

FIG. 2. Examples of tumors with or without praxis involvement (as evaluated with preoperative MRI). A: Representative axial post-Gd T1weighted image (left) of a nondominant, presumptive high-grade glioma located in the mesial parietal lobe. The tumor mass (target of resection) is located in the mesial portion of the postcentral gyrus, extends toward the parietal lobe, and has limited involvement of the praxis network. The histological diagnosis was grade IV IDH1-wild-type glioblastoma. Representative preoperative axial FLAIR image (right) of a frontal, presumptive lower-grade glioma located in the ventral premotor area (target of resection), showing extensive infiltration into the white matter and possible involvement of the anterior portion of the praxis network. The histological diagnosis was grade II IDH1-mutated astrocytoma without chromosome 1p/19q codeletion. B: Model of the praxis network, reported in an MNI model. The cortical (left) and subcortical (right) areas involved with the praxis network are highlighted in green, to serve as a reference for establishing possible praxis network involvement. Figure is available in color online only.

\section{Variables Considered for Analysis}

Clinical, imaging, and tumor variables were analyzed. Clinical variables included age, sex, seizure history and control, presence of preoperative motor deficit (evaluated with MRC grade), presence of preoperative hand apraxia, and previous treatment (surgery, chemotherapy, radiotherapy).

Imaging variables included tumor volume, lobe, praxis network involvement, and abnormalities on DWI. Tumor volume was evaluated on volumetric FLAIR images for noncontrast-enhancing lesions and on volumetric postGd T1-weighted images for contrast-enhancing lesions. Praxis network involvement was defined on preoperative MRI (T1-weighted imaging for enhancing lesion or FLAIR for nonenhancing lesion) as tumor infiltration of the white matter a) under the premotor and supplementary motor area (SMA) in the frontal lobe and/or b) under the inferior parietal lobule/primary sensitive area, where 

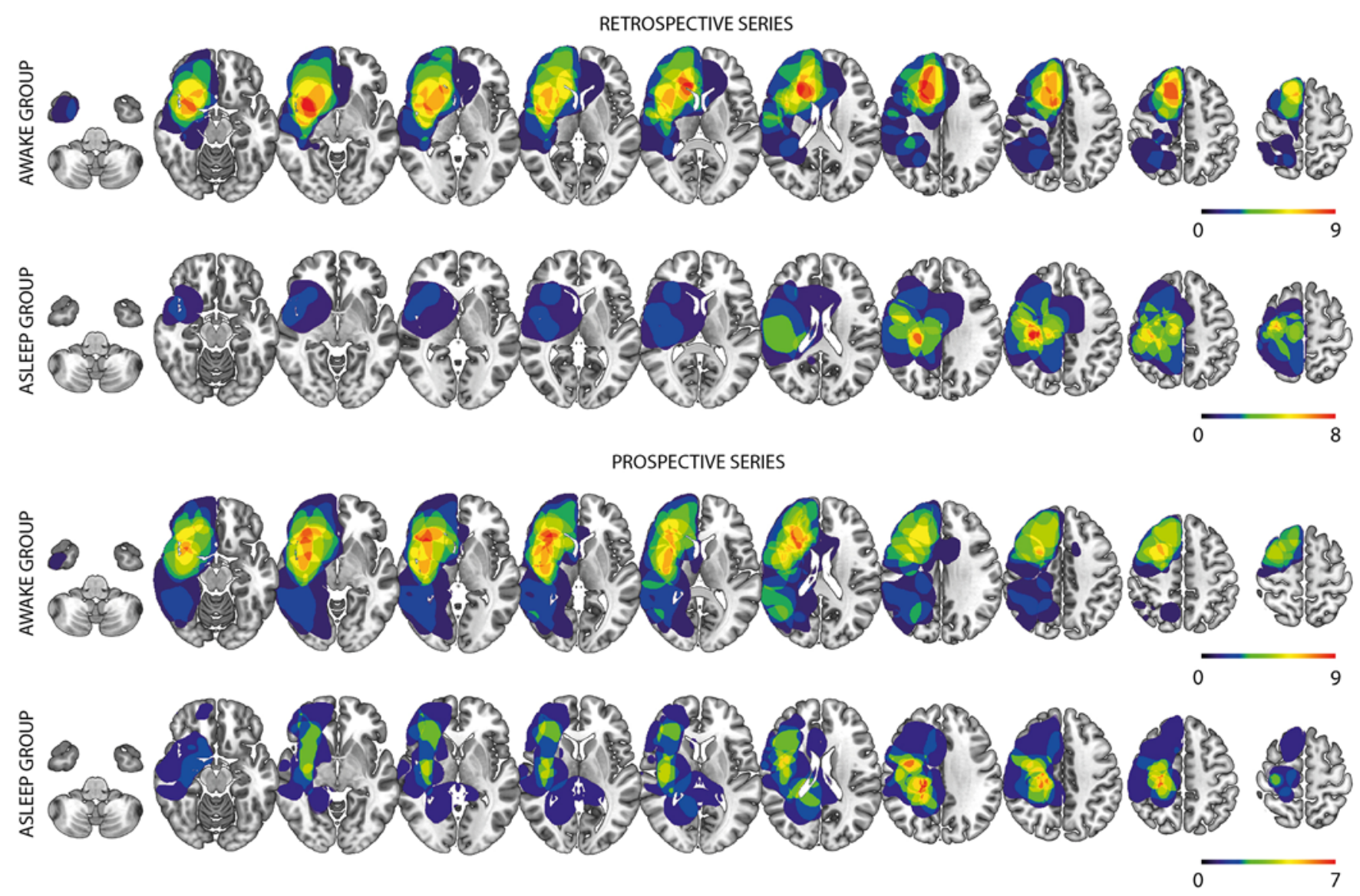

FIG. 3. Spatial analysis of tumor location. The tumor maps of the patients who underwent asleep motor mapping or awake motor mapping in the retrospective or prospective series were grouped together. Patients in the awake motor mapping group had tumor mainly in the frontal and/or parietal areas and involvement of the praxis network to a variable extent (premotor cortex, primary sensitive area, inferior parietal lobe, and underlying white matter). Patients in the asleep motor mapping group had tumor mainly in the pre- and/or postcentral gyrus, with limited involvement of the praxis network. Figure is available in color online only.

the praxis circuits are generally located (Fig. 2). ${ }^{7,8,13,14,16,21}$ Ischemia was evaluated on postoperative DWI. DWI restriction was categorized as present when $>2 \mathrm{~cm}^{3}$. The location of the abnormality on DWI and involvement of eloquent subcortical sites for motor control were documented. A subcortical site was considered eloquent when located within the course of a tract mediating motor or praxis functions. Tumor variables included histomolecular diagnosis and tumor grade according to the 2016 WHO classification. ${ }^{24}$

\section{Spatial Analysis of Tumor Location}

Preoperative T1-weighted and FLAIR images of each patient were coregistered to the standard Montreal Neurological Institute (MNI) template by using the enantiomorphic normalization process of the MR-Segment-Normalize function of the Clinical Toolbox of SPM12. The reliability of the normalization process was visually inspected on a case-by-case basis and corrected if needed. Tumor segmentation was then performed on coregistered Gd-enhanced MR images for high-grade glioma, or coregistered FLAIR images for low-grade glioma, with semiau- tomated segmentation software (ITK-SNAP). Segmented lesions were grouped by approach (asleep or awake). The amount of overlap among lesions was evaluated by producing a map of the tumor locations. The two groups were compared by creating two overlapping maps on the MNI template (MRIcron software) (Fig. 3).

\section{Statistical Analysis}

A scoring system was built to predict use of the awake motor mapping approach on the basis of clinical and imaging factors that were statistically associated with the awake approach, as defined by the analysis of the retrospective series (Fig. 4A and B). A specific weight was given to each significant factor on the basis of both the statistical and clinical data analysis. The ability of the scoring system and the best cutoff value were then tested with receiver operating characteristic analysis and Youden's statistic in order to design the "motor mapping score" (Fig. 4C). The chi-square and Fisher exact tests were used to evaluate the associations among factors. The exact McNemar's test was used for matched comparisons. No multivariable models were fitted because of the descriptive nature of this study. 

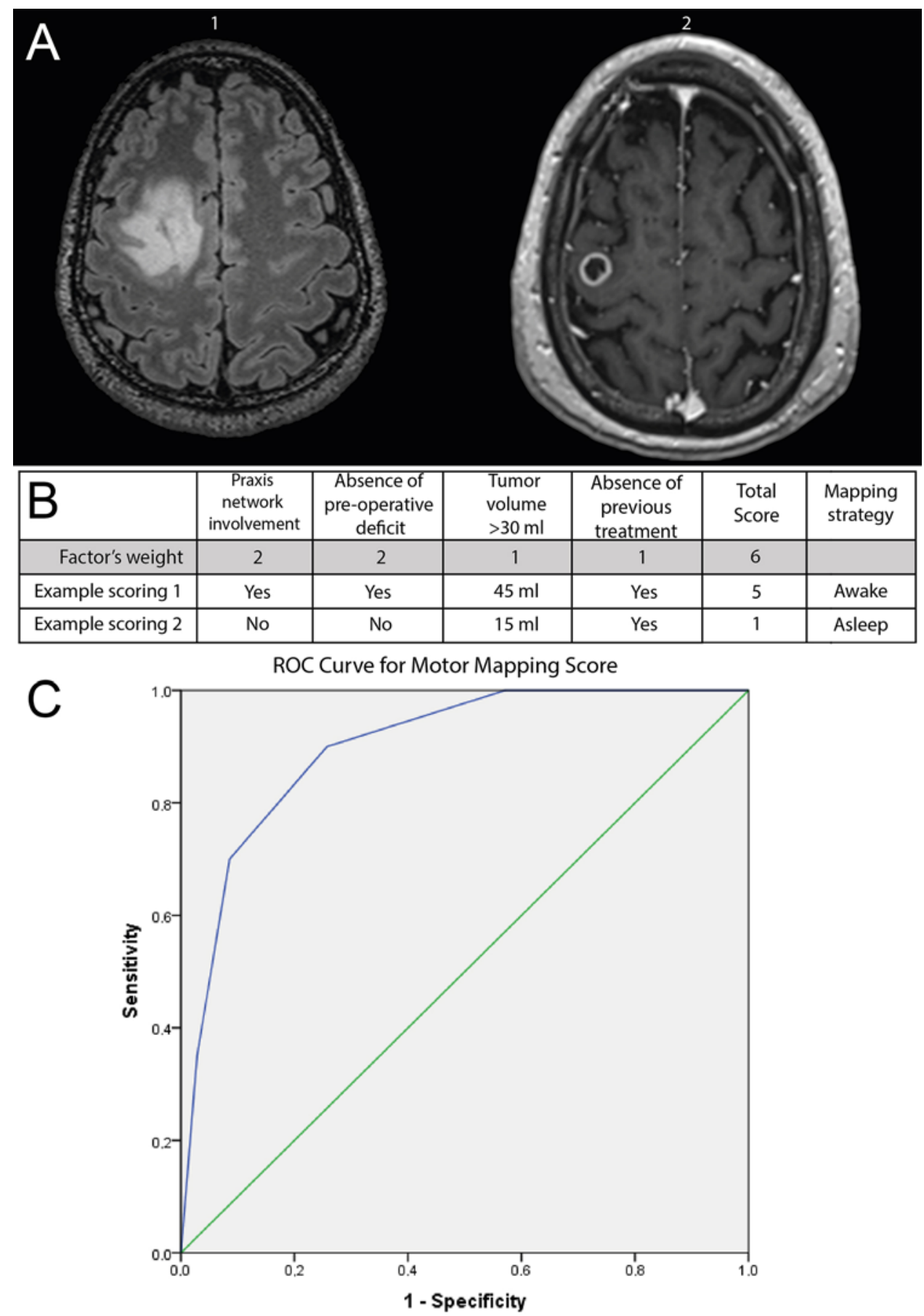

FIG. 4. Examples of the motor mapping score. A: Two examples of tumor in the frontal lobe. Representative axial FLAIR image (left) of a presumptive lower-grade glioma involving the dorsal premotor area. To determine the motor mapping score, the tumor volume, presumptive involvement of the praxis network, and clinical and neurological condition of the patient were evaluated. Tumor volume was measured on volumetric FLAIR with semiautomatic segmentation (iPlan, Brainlab). The volume was $45 \mathrm{~cm}^{3}$ (score $=1$ ). The patient had no motor deficit (score $=2$ ) or previous treatment (score $=1)$. Analysis of the preoperative MR images and model of the praxis network indicated involvement of the praxis network (score $=2$ ). The total score was 6 , supporting use of the awake motor mapping protocol. Representative axial post-Gd T1-weighted image (right) of a presumptive high-grade glioma involving the precentral gyrus. To determine the motor mapping score, the tumor volume, presumptive involvement of the praxis network, and clinical and neurological condition of the patient were evaluated. Tumor volume was measure on volumetric post-Gd T1-weighted images with semiautomatic segmentation (iPlan, Brainlab). Tumor volume was $15 \mathrm{~cm}^{3}$ (score $\left.=0\right)$. At admission, the patient had mild paresis $(4 / 5 \mathrm{MRC}$ grade) involving the right hand $($ score $=0)$. No previous treatment was reported (score $=1$ ). Analysis of the preoperative MR images and model of the praxis network did not indicate involvement of the praxis network (score $=0$ ). The total score was 1 , supporting use of the asleep motor mapping protocol. B: Motor mapping scores of these 2 patients. Score $>3$ favors adoption of the awake motor mapping protocol. C: Receiver operating characteristic (ROC) curve of the proposed motor mapping score. Figure is available in color online only. 
IBM SPSS version 24 (IBM Corp.) was used to perform the analysis.

\section{Results \\ Retrospective Series}

Patients

Of 352 patients with nondominant tumors located within $2 \mathrm{~cm}$ of the central sulcus, 135 patients fulfilled the criteria. Clinical and imaging features are shown in Table 1. Sixty-nine patients were treated under general anesthesia (asleep group) and 66 under asleep-awake-anesthesia (awake group).

\section{Preoperative Clinical Variables and Mapping Strategy}

The two groups differed in terms of preoperative motor deficits, seizure control, and previous treatments (Table 2). Fewer patients in the awake group had preoperative motor deficits (58 [88\%] had 5/5 MRC grade deficit vs 54 [78\%] in the asleep group, $\mathrm{p}=0.003$ ), but more patients in the asleep group had poor seizure control than patients in the awake group (19 [44\%] vs 5 [10\%], respectively; $\mathrm{p}=$ $0.023)$ or had received radiotherapy $(11[16 \%]$ vs 3 [5\%], $p$ $=0.012)$ or undergone previous surgery $(51 \%$ vs $24 \%, \mathrm{p}=$ $0.002)$. Other clinical factors, and particularly the presence of preoperative hand apraxia, were comparable.

\section{Imaging Variables and Mapping Strategy}

Preoperative tumor volume was greater in the awake group than in the asleep group (median $39.7 \mathrm{~cm}^{3}$ vs 16.1 $\mathrm{cm}^{3}$, respectively; $\mathrm{p}<0.001$ ) (Table 2). A greater percentage of patients in the awake group (86\%) had involvement of the praxis network than the asleep group (15\%) (p < $0.001)$. In the asleep group, tumor was mainly located in the precentral gyrus and/or its descending pathways, but involvement of the premotor or postcentral areas was limited. In 27 (20\%) patients, the SMA was involved. In the awake group, tumor was mostly located in premotor areas, in either dorsomedial prefrontal white matter or white/ gray matter toward the insula or basal ganglia (Fig. 3).

\section{Functional Outcome and Mapping Strategy}

Perioperative morbidity was low and comparable between the two groups (1 patient died in each group, $\mathrm{p}=$ 0.545 ). Patients with a previous motor (strength) deficit who underwent awake operations experienced difficulties performing hMT during surgery, and this interfered with our ability to identify the praxis circuits. No operative deaths were recorded.

Functional outcome was evaluated in terms of motor strength and apraxia, immediately and at follow-up at 1-3 months. Immediately after surgery, the rates of apraxia were comparable between the two groups (12\% of awake group and $19 \%$ of asleep group, $\mathrm{p}=0.2822$ ) (Table 2), whereas the proportion of patients with severe strength deficit was greater in the asleep group ( $28 \%$ of patients had $\leq 3 / 5 \mathrm{MRC}$ grade deficit) than the awake group $(14 \%)(\mathrm{p}=0.005)(\mathrm{Ta}-$ ble 2). SMA syndrome developed in $2(18 \%)$ patients in the asleep group and $2(14 \%)$ patients in the awake group. Most deficits resolved at 1-3 months after surgery, and the proportions of patients with permanent strength deficits $(4 \%$ of awake group vs $0 \%$ of asleep group) or apraxia (6\% of awake group vs $12 \%$ of asleep group) were comparable. However, patients with tumor larger than $30 \mathrm{~cm}^{3}$ involving the praxis network who underwent asleep motor mapping had a higher rate of permanent apraxia $(18.75 \%)$.

Rates of postoperative seizure control were also similar, and most patients were seizure free (Engel class I). A greater proportion of patients in the awake group had abnormalities on immediately postoperative DWI scans than patients in the asleep group ( $48 \%$ vs $16 \%, \mathrm{p}=0.001)$. Most of these alterations were small $\left(<1 \mathrm{~cm}^{3}\right)$, were located in both eloquent and noneloquent areas, and resolved on subsequent MR images.

\section{Oncological Outcome and Mapping Strategy}

EOR, when expressed in terms of percentage of resected tumor, was similar between groups (mean EOR $94 \%$ for the awake group vs $96 \%$ for the asleep group, $\mathrm{p}=$ 0.254 ); complete resection was achieved in $82 \%$ of patients in the asleep group versus $64 \%$ of the awake group (Table 2). However, RTV was larger in patients in the awake group than in patients in the asleep group (mean $3.7 \mathrm{~cm}^{3} \mathrm{vs}$ $1.16 \mathrm{~cm}^{3}$, respectively; $p=0.029$ ), which is consistent with the fact that preoperative tumor volume was greater in the awake group. Subtotal resection was documented in $36 \%$ of patients in the awake group and $18 \%$ of patients in the asleep group. Identification of primary motor fibers and/or praxis circuits was the main reason for stopping resection.

\section{Tumor Variables and Mapping Strategy}

Tumor grade and histomolecular diagnosis were comparable (Table 2). Low-grade tumor was diagnosed in 53\% of patients in the awake group and in 55\% of patients in the asleep group.

\section{Motor Mapping Score}

Absence of preoperative motor deficit, absence of previous treatment (radiotherapy and surgery), tumor volume $\left(>30 \mathrm{~cm}^{3}\right)$, and involvement of the praxis network were associated with the use of awake motor mapping and used to design a predictive mapping score for the use of awake motor mapping. On the basis of the intraoperative findings, clinical outcome data generated from our analysis of the retrospective series, and previously published evidence, ${ }^{12,14}$ involvement of the praxis network, and absence of preoperative motor deficits were weighted as highly relevant variables $($ score $=2)$; the other two variables, i.e., tumor volume $\left(>30 \mathrm{~cm}^{3}\right)$ and absence of previous treatment, were weighted as moderately relevant variables (score $=1$ ). The absence of any of these variables was scored as 0 . An example of the scoring system is shown in Fig. 4A and B. The discriminative power of the score was validated in a prospective series with receiver operating characteristic analysis, resulting in a high discriminative value (area under the curve $=0.890 ; 90 \%$ sensitivity; $74.3 \%$ specificity) (Fig. 4C). The best cutoff value to discriminate use of the awake procedure was $>3$, as calculated with Youden's index.

\section{Prospective Series}

Patients

Fifty-two patients were prospectively enrolled in this 
TABLE 2. Clinical and imaging variables of the patients included in the retrospective series according to the adopted anesthesia mapping protocol

\begin{tabular}{|c|c|c|c|}
\hline Variable & $\begin{array}{l}\text { Awake Group } \\
\quad(n=66)\end{array}$ & $\begin{array}{l}\text { Asleep Group } \\
\quad(n=69)\end{array}$ & $\mathrm{p}$ Value \\
\hline \multicolumn{4}{|l|}{ Preop } \\
\hline \multicolumn{4}{|l|}{ Sex } \\
\hline Male & $31(47)$ & $42(61)$ & \multirow{2}{*}{-0.105} \\
\hline Female & $35(53)$ & $27(39)$ & \\
\hline Age, mean (range), yrs & $41.9(18-68)$ & $45.5(18-75)$ & 0.119 \\
\hline \multicolumn{4}{|l|}{ 1st surgery } \\
\hline Yes & $50(76)$ & $34(49)$ & \multirow{2}{*}{-0.002} \\
\hline No & $16(24)$ & $35(51)$ & \\
\hline RT & $3(5)$ & $11(16)$ & 0.012 \\
\hline \multicolumn{4}{|l|}{ Seizure } \\
\hline Yes & $49(75)$ & $58(84)$ & \multirow{2}{*}{-0.160} \\
\hline No & $17(25)$ & $11(16)$ & \\
\hline \multicolumn{4}{|l|}{ Seizure control } \\
\hline Yes & $44(90)$ & $39(66)$ & \multirow{2}{*}{0.023} \\
\hline No & $5(10)$ & $19(44)$ & \\
\hline \multicolumn{4}{|l|}{ MRC grade } \\
\hline $5 / 5$ & $58(88)$ & $54(78)$ & \multirow{3}{*}{0.003} \\
\hline $4 / 5$ & $5(8)$ & $13(19)$ & \\
\hline$\leq 3 / 5$ & $3(4)$ & $2(3)$ & \\
\hline Hand apraxia & $2(3)$ & $1(1)$ & 0.501 \\
\hline \multicolumn{4}{|l|}{ Lobe } \\
\hline Frontal & $51(77)$ & $55(80)$ & \multirow{4}{*}{-0.051} \\
\hline Parietal & $9(13)$ & $9(13)$ & \\
\hline Frontoparietal & $1(2)$ & $5(7)$ & \\
\hline Other & $5(8)$ & $0(0)$ & \\
\hline \multicolumn{4}{|l|}{ Tumor vol, $\mathrm{cm}^{3}$} \\
\hline Mean (range) & $39.7(1.7-159.6)$ & $16.1(1.4-149.8)$ & \multirow{2}{*}{$-<0.001$} \\
\hline Median & 30.98 & 12.35 & \\
\hline \multicolumn{4}{|l|}{$\begin{array}{l}\text { Praxis network } \\
\text { involvement }\end{array}$} \\
\hline Yes & $57(86)$ & $10(15)$ & \multirow{2}{*}{$-<0.001$} \\
\hline No & $9(14)$ & $59(85)$ & \\
\hline \multicolumn{4}{|l|}{ SMA involvement } \\
\hline Yes & $14(21)$ & $13(20)$ & \multirow{2}{*}{0.731} \\
\hline No & $52(79)$ & $56(80)$ & \\
\hline Periop morbidity* & $1(1)$ & $1(1)$ & 0.545 \\
\hline \multicolumn{4}{|l|}{ Postop } \\
\hline \multicolumn{4}{|l|}{ MRC grade } \\
\hline \multicolumn{4}{|l|}{ At 5 days } \\
\hline $5 / 5$ & $34(52)$ & $20(29)$ & \multirow{3}{*}{0.005} \\
\hline $4 / 5$ & $23(35)$ & $30(43)$ & \\
\hline$\leq 3 / 5$ & $9(14)$ & $19(28)$ & \\
\hline \multicolumn{4}{|l|}{ At $1-3$ mos } \\
\hline $5 / 5$ & $50(76)$ & $60(87)$ & \\
\hline $4 / 5$ & $13(20)$ & $9(13)$ & 0.504 \\
\hline$\leq 3 / 5$ & $3(4)$ & $0(0)$ & \\
\hline
\end{tabular}

CONTINUED IN NEXT COLUMN »
» CONTINUED FROM PREVIOUS COLUMN

TABLE 2. Clinical and imaging variables of the patients included in the retrospective series according to the adopted anesthesia mapping protocol

\begin{tabular}{|c|c|c|c|}
\hline Variable & $\begin{array}{c}\text { Awake Group } \\
(n=66)\end{array}$ & $\begin{array}{l}\text { Asleep Group } \\
\quad(n=69)\end{array}$ & $\mathrm{p}$ Value \\
\hline \multicolumn{4}{|l|}{ Postop (continued) } \\
\hline \multicolumn{4}{|l|}{ Apraxia } \\
\hline \multicolumn{4}{|l|}{ At 5 days } \\
\hline Yes & $8(12)$ & $13(19)$ & \multirow{2}{*}{-0.2822} \\
\hline No & $58(88)$ & $56(81)$ & \\
\hline \multicolumn{4}{|l|}{ At $1-3$ mos } \\
\hline Yes & $4(6)$ & $8(12)$ & \multirow{2}{*}{-0.2379} \\
\hline No & $62(94)$ & $61(88)$ & \\
\hline \multicolumn{4}{|l|}{ SMA syndrome } \\
\hline Yes & $2(14)$ & $2(18)$ & \multirow{2}{*}{-0.560} \\
\hline No & $12(86)$ & $11(82)$ & \\
\hline \multicolumn{4}{|l|}{ Engel class } \\
\hline I & $54(82)$ & $43(62)$ & \multirow{4}{*}{0.053} \\
\hline II & $11(17)$ & $22(32)$ & \\
\hline III & $0(0)$ & $3(4)$ & \\
\hline IV & $1(1)$ & $1(2)$ & \\
\hline \multicolumn{4}{|l|}{$\mathrm{RTV}, \mathrm{cm}^{3}$} \\
\hline Mean (range) & $3.7(0-53.86)$ & $1.16(0-29)$ & \multirow{2}{*}{0.029} \\
\hline Median & 0 & 0 & \\
\hline \multicolumn{4}{|l|}{ EOR } \\
\hline Mean, \% & 94 & 96 & 0.254 \\
\hline $100 \%$ & $42(64)$ & $57(82)$ & \\
\hline $90-99 \%$ & $10(15)$ & $6(9)$ & \\
\hline$<90 \%$ & $14(21)$ & $6(9)$ & 0.071 \\
\hline \multicolumn{4}{|l|}{ Alteration on DWI } \\
\hline Eloquent site & $14(21)$ & $6(9)$ & \multirow{2}{*}{0.001} \\
\hline Not eloquent site & $18(27)$ & $5(7)$ & \\
\hline \multicolumn{4}{|l|}{ Histologic diagnosis } \\
\hline LGG & $35(53)$ & $38(55)$ & \multirow{3}{*}{0.808} \\
\hline$H G G$ & $25(38)$ & $23(33)$ & \\
\hline Other† & $6(9)$ & $8(12)$ & \\
\hline \multicolumn{4}{|l|}{ RT } \\
\hline Yes & $29(44)$ & $33(48)$ & \multirow{2}{*}{0.329} \\
\hline No & $37(56)$ & $36(52)$ & \\
\hline
\end{tabular}

Values are shown as number (percent) unless indicated otherwise. Boldface type indicates statistical significance $(p<0.05)$.

* One patient died of pulmonary embolism, and 1 patient completely recovered from a postoperative hematoma that was immediately evacuated.

† Includes 8 patients with metastasis, 4 with cortical dysplasia, 2 with cavernous malformation, and 2 with gliosis. 
series. Clinical and imaging features are shown in Table 1. For each patient, the four variables (see Motor Mapping Score) were evaluated and scored. Patients underwent awake or asleep operations according to the indication of the mapping score: 17 were treated while asleep because their score was $<3$, and 35 were treated while awake because their score was $>3$ (Table 3 ). The map of tumor locations showed that patients in the awake group had larger tumor volume and tumor involving the frontal and/or parietal lobe; only 1 patient had SMA involvement. Patients in the asleep group had smaller tumor and tumor involving the precentral gyrus, and SMA involvement was very limited (1 patient) (Fig. 3). Three patients in the awake group and 2 in the asleep group were "unmatched" for at least one criterion of the motor mapping score, and these patients were treated with a different motor mapping protocol than that indicated by the motor mapping score.

\section{Functional Results}

The proportions of patients with immediate motor deficits were comparable between groups (46\% of patients in the awake group vs $24 \%$ of the asleep group), as were the proportions of patients with permanent deficits $(12 \%$ of patients in the awake and asleep groups; $3 \%$ of patients in the awake group had severe permanent deficits vs $0 \%$ of the asleep group) (Table 3). No patients developed SMA syndrome. Praxis deficits were observed immediately after surgery in $14 \%$ of patients in the awake group and $18 \%$ of patients in the asleep group; no permanent apraxia developed in the asleep group, and only 2 patients in the awake group developed permanent apraxia (Table 3). Seizure control was comparable and high in both groups.

Small alterations were recorded on immediately postoperative DWI scans in both groups (29\% of patients in the awake group vs $35 \%$ of the asleep group), and most resolved in 3 months (Table 3 ).

\section{Oncological and Histomolecular Results}

The median EOR was $97 \%$ in both groups. Complete resection was achieved in $69 \%$ of patients in the awake group versus $76 \%$ of those in the asleep group, without statistical difference. The median RTV was $0 \mathrm{~cm}^{3}$ in both the asleep and awake groups, with greater mean RTV in the awake group $\left(1.8 \mathrm{~cm}^{3}\right.$ vs $\left.0.6 \mathrm{~cm}^{3}\right)$ because of the larger preoperative tumor volume, but without statistical difference (Table 3). The histomolecular diagnoses were similar between groups (Table 3 ).

\section{Clinical and Imaging Features of the Unmatched Patients}

Details are reported in Table 4. Three patients without involvement of the praxis network who underwent awake operations had presumptive lower-grade glioma. For these patients, the intention of treatment was to achieve supratotal resection, ${ }^{25}$ and therefore intraoperative testing of the praxis and cognitive networks was relevant because these networks were close to the planned limit of resection on preoperative MRI. Two patients underwent asleep operations despite involvement of the praxis network and indication for awake mapping based on the mapping score. Both patients refused to undergo awake operation, and both developed mild praxis deficit that was persistent at postsurgical evaluation at 1-3 months.

\section{Discussion}

Preservation of motor function relies on intraoperative identification of areas and pathways involved in ideation, planning, execution, and control of movements. ${ }^{4,14,17}$ The available neurophysiological paradigms (LF and HF stimulation) allow the surgeon to precisely recognize the primary motor cortex and its descending fibers in patients under both asleep and awake anesthesia. ${ }^{4} \mathrm{~A}$ patient with a tumor in the dominant hemisphere would probably undergo awake surgery in an effort to preserve language and cognitive functions; however, for a patient with a lesion in the nondominant hemisphere, the choice of the anesthesia regimen and motor mapping strategy is, at the moment, based on surgeon preference. ${ }^{22}$ Generally, there is no consensus regarding the best anesthesia and mapping strategy to apply to routine clinical practice. The choice of the anesthesia regimen depends on various factors, such as the patient's clinical and neurological condition, tumor size and location, type of neurophysiological paradigm, anesthesiological constraints, and surgeon preference/experience. ${ }^{17,22}$ In recent years, we have operated on several patients with tumors involving the motor pathways by using either an awake or asleep motor mapping strategy. ${ }^{12,14}$ These patients had tumors involving motor areas and pathways to variable extent and had various clinical conditions. From this past work, we learned the importance of adapting the neurophysiological paradigm to the clinical context in order to enhance resection and decrease postoperative motor (strength) morbidity. ${ }^{1,12}$ Similarly, our work also questioned the clinical relevance of extending mapping to nonprimary motor areas and circuits ${ }^{14}$ to avoid the occurrence of apraxia.

In the present study, we initially reviewed the strategies applied to patients with tumor in the nondominant hemisphere, with the aim of identifying which clinical and imaging factors are better correlated with motor and oncological outcomes in this specific set of patients. We restricted the analysis to patients with tumors located within $2 \mathrm{~cm}$ in front of or behind the central sulcus and involving M1, its originating fibers, and the praxis network. Motor outcome was analyzed in terms of motor strength and praxis, and oncological outcome was analyzed in terms of EOR. Ideally, the best protocol, according to clinical context, should provide patients with no additional motor deficits and extensive tumor resection.

Among the variables analyzed, the presence of a previous motor (strength) deficit, previous treatment (surgery and/or radiotherapy), large tumor volume $\left(>30 \mathrm{~cm}^{3}\right)$, and possible anatomical involvement of the tumor in the praxis network on preoperative MR images were associated with asleep or awake motor mapping. We designed a motor mapping score by combining these factors, and each variable was given a score based on the findings of the retrospective series and previous data generated by our group. Involvement of the praxis network and absence of preoperative motor deficits were weighted as highly relevant variables (score $=2$ ) that favor use of awake motor map- 
TABLE 3. Clinical and imaging variables of patients enrolled in the prospective series according to adopted anesthesia mapping protocol

\begin{tabular}{|c|c|c|c|}
\hline Variable & $\begin{array}{c}\text { Awake Group } \\
\quad(n=35)\end{array}$ & $\begin{array}{l}\text { Asleep Group } \\
\quad(n=17)\end{array}$ & $\mathrm{p}$ Value \\
\hline \multicolumn{4}{|l|}{ Preop } \\
\hline \multicolumn{4}{|l|}{ Sex } \\
\hline Male & $21(60)$ & $13(77)$ & \multirow{2}{*}{0.242} \\
\hline Female & $14(40)$ & $4(23)$ & \\
\hline Age, mean (range), yrs & $40.7(25-63)$ & $57.0(50-62)$ & $<0.001$ \\
\hline \multicolumn{4}{|l|}{ 1st surgery } \\
\hline Yes & $22(63)$ & $9(53)$ & \multirow{2}{*}{0.494} \\
\hline No & $9(37)$ & $8(47)$ & \\
\hline \multicolumn{4}{|l|}{ RT } \\
\hline Yes & $6(17)$ & $3(18)$ & \multirow{2}{*}{0.652} \\
\hline No & $29(83)$ & $14(82)$ & \\
\hline \multicolumn{4}{|l|}{ Seizure } \\
\hline Yes & $27(77)$ & $14(82)$ & \multirow{2}{*}{0.768} \\
\hline No & $8(23)$ & $3(18)$ & \\
\hline \multicolumn{4}{|l|}{ Seizure control } \\
\hline Yes & $24(88)$ & $11(79)$ & \multirow{2}{*}{0.388} \\
\hline No & $3(12)$ & $3(21)$ & \\
\hline \multicolumn{4}{|l|}{ MRC grade } \\
\hline $5 / 5$ & $35(100)$ & $14(82)$ & \multirow{3}{*}{0.010} \\
\hline $4 / 5$ & $0(0)$ & $3(18)$ & \\
\hline$\leq 3 / 5$ & $0(0)$ & $0(0)$ & \\
\hline \multicolumn{4}{|l|}{ Hand apraxia } \\
\hline Yes & $0(0)$ & $1(2)$ & \multirow{2}{*}{0.457} \\
\hline No & $35(100)$ & $16(98)$ & \\
\hline \multicolumn{4}{|l|}{ Lobe } \\
\hline Frontal & $25(71)$ & $9(53)$ & \multirow{4}{*}{0.079} \\
\hline Parietal & $10(29)$ & $5(29)$ & \\
\hline Frontoparietal & $0(0)$ & $1(6)$ & \\
\hline Other & $0(0)$ & $2(12)$ & \\
\hline \multicolumn{4}{|l|}{ Tumor vol, $\mathrm{cm}^{3}$} \\
\hline Mean (range) & $56.7(1-229)$ & $31.9(2-80)$ & \multirow{2}{*}{0.020} \\
\hline Median & 14.6 & 23.5 & \\
\hline \multicolumn{4}{|l|}{$\begin{array}{l}\text { Praxis network } \\
\text { involvement }\end{array}$} \\
\hline Yes & $27(77)$ & $3(16)$ & \multirow{2}{*}{0.003} \\
\hline No & $8(23)$ & $14(84)$ & \\
\hline \multicolumn{4}{|l|}{ SMA involvement } \\
\hline Yes & $1(3)$ & $1(6)$ & \multirow{2}{*}{0.595} \\
\hline No & $34(97)$ & $16(94)$ & \\
\hline Periop morbidity* & $1(3)$ & $0(0)$ & 0.389 \\
\hline \multicolumn{4}{|l|}{ Postop } \\
\hline MRC grade & & & \\
\hline At 5 days & & & \\
\hline $5 / 5$ & $19(54)$ & $13(76)$ & \\
\hline $4 / 5$ & $9(26)$ & $3(18)$ & 0.257 \\
\hline$\leq 3 / 5$ & $7(20)$ & $1(6)$ & \\
\hline
\end{tabular}

» CONTINUED FROM PREVIOUS COLUMN

TABLE 3. Clinical and imaging variables of patients enrolled in the prospective series according to adopted anesthesia mapping protocol

\begin{tabular}{|c|c|c|c|}
\hline Variable & $\begin{array}{c}\text { Awake Group } \\
(n=35)\end{array}$ & $\begin{array}{l}\text { Asleep Group } \\
\quad(n=17)\end{array}$ & $\mathrm{p}$ Value \\
\hline \multicolumn{4}{|c|}{ MRC grade (continued) } \\
\hline \multicolumn{4}{|l|}{ At $1-3$ mos } \\
\hline $5 / 5$ & $31(88)$ & $15(88)$ & \multirow{3}{*}{0.738} \\
\hline $4 / 5$ & $3(9)$ & $2(12)$ & \\
\hline$\leq 3 / 5$ & $1(3)$ & $0(0)$ & \\
\hline \multicolumn{4}{|l|}{ Apraxia } \\
\hline \multicolumn{4}{|l|}{ At 5 days } \\
\hline Yes & $5(14)$ & $3(18)$ & \multirow{2}{*}{0.753} \\
\hline No & $30(86)$ & $14(82)$ & \\
\hline \multicolumn{4}{|l|}{ At $1-3$ mos } \\
\hline Yes & $2(6)$ & $0(0)$ & \multirow{2}{*}{0.315} \\
\hline No & $33(94)$ & $17(100)$ & \\
\hline \multicolumn{4}{|l|}{ SMA syndrome } \\
\hline Yes & $0(0)$ & $0(0)$ & \multirow{2}{*}{$\mathrm{NA}$} \\
\hline No & $1(100)$ & $1(100)$ & \\
\hline \multicolumn{4}{|l|}{ Engel class } \\
\hline I & $31(89)$ & $17(0)$ & \multirow{4}{*}{0.207} \\
\hline II & $4(11)$ & $0(0)$ & \\
\hline III & $0(0)$ & $0(0)$ & \\
\hline IV & $0(0)$ & $0(0)$ & \\
\hline \multicolumn{4}{|l|}{$\mathrm{RTV}, \mathrm{cm}^{3}$} \\
\hline Mean (range) & $1.8(0-19.3)$ & $0.6(0-4.2)$ & \multirow{2}{*}{0.138} \\
\hline Median & 0 & 0 & \\
\hline \multicolumn{4}{|l|}{ EOR } \\
\hline Mean, \% & 97 & 97 & 0.841 \\
\hline $100 \%$ & $24(69)$ & $13(76)$ & \\
\hline $90-99 \%$ & $9(26)$ & $4(23)$ & \\
\hline$<90 \%$ & $2(5)$ & $0(0)$ & 0.530 \\
\hline \multicolumn{4}{|l|}{ Alteration on DWI } \\
\hline Eloquent site & $2(6)$ & $1(5)$ & \multirow{3}{*}{0.368} \\
\hline Not eloquent site & $8(23)$ & $5(30)$ & \\
\hline No & $25(71)$ & $11(65)$ & \\
\hline \multicolumn{4}{|l|}{ Histologic diagnosis } \\
\hline LGG & $26(74)$ & $8(47)$ & \multirow{3}{*}{0.043} \\
\hline HGG & $7(20)$ & $9(53)$ & \\
\hline Other† & $2(6)$ & $0(0)$ & \\
\hline \multicolumn{4}{|l|}{ RT } \\
\hline Yes & $11(32)$ & $6(35)$ & \multirow{2}{*}{0.834} \\
\hline No & $24(68)$ & $11(65)$ & \\
\hline
\end{tabular}

$\mathrm{NA}=$ not applicable.

Values are shown as number (percent) unless indicated otherwise. Boldface type indicates statistical significance $(p<0.05)$.

* Patient died of status epilepticus.

† Includes 2 patients with breast metastasis and 1 with ganglioglioma. 


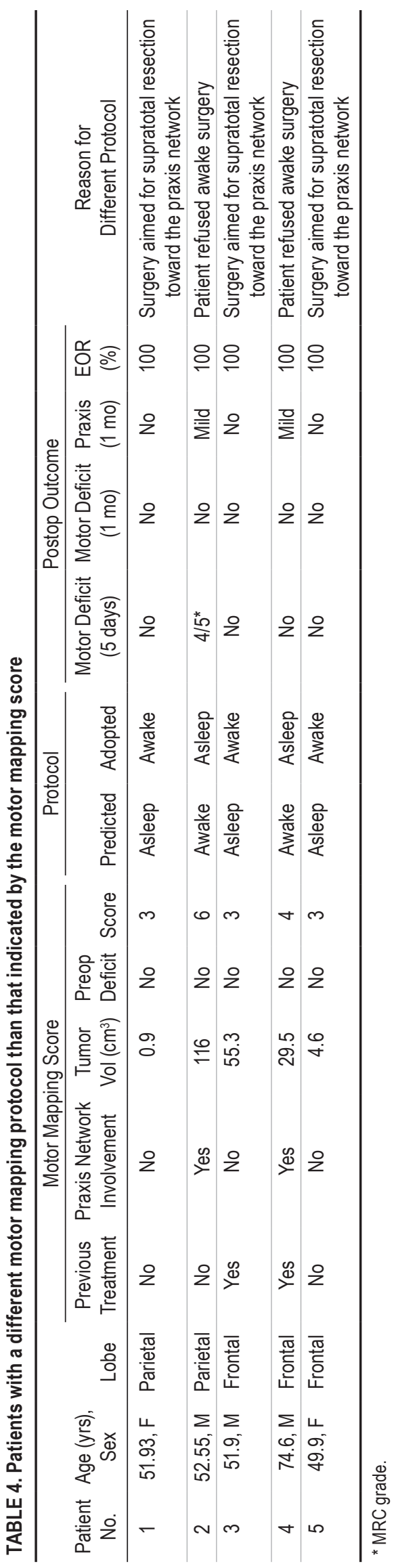

ping. Involvement of the praxis network is associated with the need to map and preserve the praxis circuits in order to avoid the occurrence of apraxia, which is very difficult to treat and severely impairs patients' quality of life. ${ }^{14} \mathrm{In}$ the retrospective series, patients with tumors involving the praxis network who underwent operations with the asleep mapping protocol developed permanent apraxia. In addition, the existence of a previous strength deficit interfered with proper execution of the praxis task, making its evaluation difficult in the awake setting and interfering with our ability to identify and preserve the praxis circuit. Large tumor volume $\left(>30 \mathrm{~cm}^{3}\right)$ and absence of previous treatment were scored as moderately relevant factors (score $=$ 1), again favoring adoption of the awake motor mapping protocol. The presence of large tumor volume and/or (consequent) involvement of the praxis network to any extent makes the use of awake anesthesia and a wide motor mapping strategy preferable, to decrease the risk of inducing motor and praxis impairment.

Previous treatment, and radiotherapy in particular, may alter the excitability of motor areas or fibers or interfere with the proper functional assets of associative motor networks, making mapping (with HF stimulation) easier in asleep patients. The best cutoff value, score $>3$, for identification of the average awake procedure was calculated with Youden's index. This score was then validated in a prospective series, in which patients with tumor located within $2 \mathrm{~cm}$ behind or above the central sulcus were admitted during a 6-month period and underwent an awake or asleep motor mapping surgical procedure according to the indication suggested by the motor mapping score. For each patient, four variables (see Motor Mapping Score) were evaluated and scored. Of 52 patients enrolled, 17 underwent asleep operations because they had a score $<3$, and 35 underwent awake operations because they had a score $>3$. The functional and oncological outcomes were comparable between groups and with those of the retrospective cohort, supporting the clinical validity of the proposed score. Very few patients were unmatched, i.e., underwent an operation with a different motor mapping approach than that suggested by the score. Analysis of these patients also highlighted the clinical limitations of the proposed score.

\section{Limitations}

The data presented here are related to the use of the HF stimulation paradigm for asleep motor mapping and the LF stimulation paradigm for awake motor mapping. HF stimulation has been shown to be the most effective paradigm to elicit motor responses in most clinical conditions and the best for use in the asleep setting. ${ }^{1}$ On the contrary, LF stimulation has been shown to have limitations in patients with altered excitability and works better in the awake setting. ${ }^{1,20}$ Consequently, when LF stimulation is the preferred paradigm or the only one available for motor mapping, the awake anesthesia regimen is recommended. ${ }^{20}$ Evaluation of tumor volume and involvement of the praxis network takes into consideration the spatial relationship between tumor location, resection margins, and the locations of the circuits subserving praxis. ${ }^{6,14}$ However, when resection larger than the visualized tumor volume 
is planned (as in supratotal resection), the circuits mediating praxis may be encountered during resection; in these cases, an awake anesthesia regimen is preferred to allow testing and preservation of praxis along with other functions. ${ }^{26,27}$ When awake motor mapping is recommended, patient preference or anesthesiological constraints may drive the strategy toward the asleep setting; in this case, the surgeon should be ready to adapt the intraoperative strategy to the clinical context. Use of HF stimulation in the asleep setting requires a complex intraoperative monitoring machine, a trained neurophysiology technician/neurophysiologist in the operating room, and an intraoperative neurosurgeon trained in neurophysiology, which may limit its widespread use in all surgical rooms.

\section{Conclusions}

Surgery for tumors located near the eloquent area for motor control is feasible, and when an appropriate mapping strategy is applied, has a low incidence of postoperative motor and praxis deficits. Asleep motor mapping with an HF paradigm is preferable for patients with lesions close to or involving the central sulcus and/or patients with preoperative strength deficit and/or history of previous treatment; when, instead, the patient has no motor deficit or previous treatment and has a lesion extending to or involving the praxis network, awake motor mapping is preferable. Clinical application of the motor mapping score should be tailored to the stimulation paradigms in use, intention to treat, patient preferences, and anesthesiological constraints.

\section{Acknowledgments}

We thank Dr. Paola Borroni for linguistic editing of the paper. This work was supported by an AIRC grant (no. G18482) awarded to Dr. Bello.

\section{References}

1. Bello L, Riva M, Fava E, et al. Tailoring neurophysiological strategies with clinical context enhances resection and safety and expands indications in gliomas involving motor pathways. Neuro Oncol. 2014;16(8):1110-1128.

2. Duffau H, Mandonnet E. The "onco-functional balance" in surgery for diffuse low-grade glioma: integrating the extent of resection with quality of life. Acta Neurochir (Wien). 2013; 155(6):951-957.

3. Hervey-Jumper SL, Berger MS. Maximizing safe resection of low- and high-grade glioma. J Neurooncol. 2016;130(2): 269-282.

4. Rossi M, Sani S, Nibali MC, et al. Mapping in low-grade glioma surgery: low- and high-frequency stimulation. Neurosurg Clin N Am. 2019;30(1):55-63.

5. Borra E, Luppino G. Large-scale temporo-parieto-frontal networks for motor and cognitive motor functions in the primate brain. Cortex. 2019;118:19-37.

6. Fornia L, Rossi M, Rabuffetti M, et al. Direct electrical stimulation of premotor areas: different effects on hand muscle activity during object manipulation. Cereb Cortex. 2020; 30(1):391-405.

7. Simone L, Fornia L, Viganò L, et al. Large scale networks for human hand-object interaction: functionally distinct roles for two premotor regions identified intraoperatively. Neuroimage. 2020;204:116215.

8. Viganò L, Fornia L, Rossi M, et al. Anatomo-functional char- acterisation of the human "hand-knob": a direct electrophysiological study. Cortex. 2019;113:239-254.

9. De Witt Hamer PC, Robles SG, Zwinderman AH, et al. Impact of intraoperative stimulation brain mapping on glioma surgery outcome: a meta-analysis. J Clin Oncol. 2012;30(20): 2559-2565.

10. Duffau H. The usefulness of the asleep-awake-asleep glioma surgery. Acta Neurochir (Wien). 2014;156(8):1493-1494.

11. Szelényi A, Senft C, Jardan M, et al. Intra-operative subcortical electrical stimulation: a comparison of two methods. Clin Neurophysiol. 2011;122(7):1470-1475.

12. Rossi M, Conti Nibali M, Viganò L, et al. Resection of tumors within the primary motor cortex using high-frequency stimulation: oncological and functional efficiency of this versatile approach based on clinical conditions. J Neurosurg. 2020;133(3):642-654.

13. Fornia L, Ferpozzi V, Montagna M, et al. Functional characterization of the left ventrolateral premotor cortex in humans: a direct electrophysiological approach. Cereb Cortex. 2018; 28(1):167-183.

14. Rossi M, Fornia L, Puglisi G, et al. Assessment of the praxis circuit in glioma surgery to reduce the incidence of postoperative and long-term apraxia: a new intraoperative test. $J$ Neurosurg. 2018;130(1):17-27.

15. Almairac F, Herbet G, Moritz-Gasser S, Duffau H. Parietal network underlying movement control: disturbances during subcortical electrostimulation. Neurosurg Rev. 2014;37(3):513-517.

16. Desmurget M, Richard N, Beuriat PA, et al. Selective inhibition of volitional hand movements after stimulation of the dorsoposterior parietal cortex in humans. Curr Biol. 2018; 28(20):3303-3309.e3.

17. Rossi M, Nibali MC, Torregrossa F, et al. Innovation in neurosurgery: the concept of cognitive mapping. World Neurosurg. 2019;131:364-370.

18. Magill ST, Han SJ, Li J, Berger MS. Resection of primary motor cortex tumors: feasibility and surgical outcomes. $J$ Neurosurg. 2018;129(4):961-972.

19. Keles GE, Lundin DA, Lamborn KR, et al. Intraoperative subcortical stimulation mapping for hemispherical perirolandic gliomas located within or adjacent to the descending motor pathways: evaluation of morbidity and assessment of functional outcome in 294 patients. J Neurosurg. 2004; 100(3):369-375.

20. Han SJ, Morshed RA, Troncon I, et al. Subcortical stimulation mapping of descending motor pathways for perirolandic gliomas: assessment of morbidity and functional outcome in 702 cases. J Neurosurg. 2018;131(1):201-208.

21. Fornia L, Puglisi G, Leonetti A, et al. Direct electrical stimulation of the premotor cortex shuts down awareness of voluntary actions. Nat Commun. 2020;11(1):705.

22. Arzoine J, Levé C, Pérez-Hick A, et al. Anesthesia management for low-grade glioma awake surgery: a European LowGrade Glioma Network survey. Acta Neurochir (Wien). 2020; 162(7):1701-1707.

23. van den Bent MJ, Wefel JS, Schiff D, et al. Response assessment in neuro-oncology (a report of the RANO group): assessment of outcome in trials of diffuse low-grade gliomas. Lancet Oncol. 2011;12(6):583-593.

24. Louis DN, Perry A, Reifenberger G, et al. The 2016 World Health Organization Classification of Tumors of the Central Nervous System: a summary. Acta Neuropathol. 2016;131(6): 803-820.

25. Rossi M, Ambrogi F, Gay L, et al. Is supratotal resection achievable in low-grade gliomas? Feasibility, putative factors, safety, and functional outcome. J Neurosurg. 2020;132(6): 1692-1705.

26. Puglisi G, Sciortino T, Rossi M, et al. Preserving executive functions in nondominant frontal lobe glioma surgery: an intraoperative tool. J Neurosurg. 2018;131(2):474-480. 
27. Puglisi G, Howells H, Sciortino T, et al. Frontal pathways in cognitive control: direct evidence from intraoperative stimulation and diffusion tractography. Brain. 2019;142(8): $2451-2465$

\section{Disclosures}

The authors report no conflict of interest concerning the materials or methods used in this study or the findings specified in this paper.

\section{Author Contributions}

Conception and design: Rossi. Acquisition of data: Rossi. Analysis and interpretation of data: Rossi, Gay, Leonetti. Drafting the article: Rossi, Puglisi, Conti Nibali, Sciortino, Bello. Critically revising the article: Rossi, Conti Nibali, Viganò, Sciortino, Zito, Bello. Reviewed submitted version of manuscript: Rossi, Viganò, Gay, Riva, Bello. Approved the final version of the manuscript on behalf of all authors: Rossi. Statistical analysis: Rossi, Puglisi. Study supervision: Bello.

\section{Correspondence}

Marco Rossi: Istituto Ortopedico Galeazzi, IRCCS, Università degli Studi di Milano, Italy. marco.rossi2@unimi.it. 